Fecha de recepción: mayo 2021 Fecha de aceptación: junio 2021 Versión final: julio 2021

\section{La investigación para el diseño y cómo enfrentar al Covid-19}

Salvador E. Valdovinos-Rodríguez ${ }^{(1)}$

Resumen: Este proyecto explora el campo de la investigación para el diseño como una de las actividades que pueden formar diseñadores gráficos con los conocimientos, habilidades y actitudes para contribuir a entender el nuevo contexto y facilitar las actividades humanas en la adaptación a la nueva realidad, nueva cultura, nuevas maneras de hacer las cosas y nuevas conexiones entre los objetos y sus significados. La investigación debe ser aprendida con estrategias didácticas que acerquen a los estudiantes al usuario y su contexto para que adquieran una visión actualizada y precisa de la evolución de la sociedad.

Palabras clave: Investigación para el diseño - enseñanza de la investigación - Covid-19 proceso de diseño - talleres de diseño.

[Resúmenes en inglés y portugués en la página 219]

(1) Doctor en Educación del Arte y el Diseño por la Universidad Concordia en Canadá. Licenciado y maestro en Diseño Gráfico por la UASLP. Profesor-investigador de tiempo completo de la UACJ. Miembro del Sistema Nacional de Investigadores. Áreas de investigación: Diseño Gráfico en la Industria.

\title{
Introducción
}

Los diseñadores gráficos como comunicadores deben dominar el lenguaje mágico que entrelaza imágenes y textos, que está siempre en evolución, como lo expresó Shaughnessy (2010): "un lenguaje idiomático, un lenguaje de claves y juegos de palabras y símbolos y alusiones, de referencias culturales e inferencias perceptivas que desafían tanto el intelecto como el ojo" (p. 18). Esta habilidad de comunicación se puede ampliar a través de la conciencia cultural, prestando atención a lo que sucede al alrededor para poder discutir cualquier cosa de manera multidimensional (Press y Cooper, 2007; Shaughnessy, 2010; Stickdorn y Schneider, 2011) y potenciar la capacidad propia y la posibilidad de responder a la diversidad de problemas que enfrentamos como ha sido la pandemia del Covid-19 y el inicio de una nueva manera de vivir en el mundo que trae consigo infinidad de retos a la sociedad. 
Estos retos provocan la pregunta, ¿cómo debemos educar a los futuros diseñadores para generar interés y comprender el nuevo escenario? El Manifiesto de Icograda Design Education 2011 responde: "El poder de pensar el futuro cercano y lejano debe ser una parte integral de la educación y la práctica del diseño a través de la investigación" (Bennet y Vulpinari, 2011, p.8). Por lo tanto, la investigación se plantea fundamental, como la habilidad que despierta esta conciencia y el poder de transformación. Esa es la motivación para explorar y comprender cómo se enseña actualmente la investigación en diseño, e identificar las mejores prácticas de educación que han contribuido a formar al diseñador y las maneras de aprender de los estudiantes de hoy. Y con esta visión proponer cambios que promuevan el conocimiento, habilidades y actitudes en los diseñadores para que contribuyan con su comunidad a la adaptación al nuevo contexto.

Los contextos contemporáneos creados por la pandemia del Covid-19 muestran una creciente complejidad de la comunicación y las tecnologías, nuevas formas de relacionarnos, nuevas conexiones culturales con los objetos y el entorno, nuevos intereses, aspiraciones y valores, nuevas maneras de hacer las cosas y una completamente nueva cotidianeidad. Esta situación exige una actualización de 360 grados, en la que los diseñadores tengan la capacidad de explorar las dimensiones sociales, económicas, ambientales y políticas, para responder con soluciones efectivas y sustentables (Rogel, Moreno y Valdovinos, 2015) a los problemas que enfrenta la sociedad.

\section{Educación de la investigación para el diseño}

Los programas de diseño gráfico en las universidades permanentemente desarrollan estrategias de aprendizaje para promover las habilidades de investigación entre educadores y estudiantes. De esta manera se ha mejorado y fortalecido la educación de la investigación para diseño gráfico en los planes de estudios, pero no ha sido suficiente. Seguimos observando prácticas de investigación entre los estudiantes que no les proporcionan una visión clara de los problemas, ni logran la suficiente empatía con el usuario y su entorno. Menos aún, proponer ideas que transformen y ofrezcan mejor calidad de vida a su comunidad. Estas estrategias educativas se pueden desarrollar con base en la comprensión de la investigación para el diseño en el proceso de diseño. Entender el papel de la investigación en el proceso de diseño; cómo los estudiantes se aproximan al usuario y descubren sus escenarios, problemas o necesidades; sobre el papel de los educadores y sus mejores prácticas en la enseñanza del diseño y de la investigación. Estas son áreas que se requiere explorar de una manera profunda, para obtener una visión de lo que los estudiantes y los educadores realmente piensan y hacen sobre la investigación en su práctica del diseño.

Este proyecto ha recopilado por ocho años mejores prácticas de educadores, así como experiencias de aprendizaje de estudiantes que conectan la investigación con prácticas de diseño innovadoras y efectivas, incluyendo la tesis doctoral (Valdovinos, 2016). Igualmente, se han explorado prácticas de profesionales del diseño en donde la investigación 
ha sido crítica durante el proceso de diseño y finalmente se ha explorado en el último año ambientes donde el Covid ha tenido más impacto y la comunicación visual ha sido protagonista en la respuesta para enfrentar los retos de informar, orientar y promover las actitudes y conductas de prevención de la sociedad (Valdovinos y Rogel, 2021).

Durante esta larga exploración se ha buscado comprender los problemas y limitaciones, así como las posibilidades y oportunidades con respecto a la enseñanza de la investigación para el diseño. Se ha investigado también cuáles son las características de la investigación para el diseño y las formas diversas que toma a lo largo de las etapas del proceso de diseño, que van de lo formal a lo informal, incluyendo la intuición y las formas un tanto individuales de aproximarse al usuario y su entorno.

Los educadores de diseño son actores determinantes en la enseñanza superior. Sus experiencias profesionales, estrategias de enseñanza, habilidades y perspectivas dan forma al plan de estudios en la educación del diseño. Las experiencias y actitudes de los profesores hacia la investigación para el diseño tienen una relación directa con los perfiles, características y estilos de enseñanza en los talleres de diseño. Se han explorado sus experiencias de instrucción, prácticas y contenido curricular con respecto a la investigación. Esta investigación ha permitido identificar no solo estrategias efectivas de enseñanza del diseño gráfico, sino también limitaciones de tales enfoques y consideraciones contextuales que son totalmente pertinentes para la experiencia que vive el mundo con esta pandemia.

El fenómeno educativo involucra la dimensión de aprendizaje de los estudiantes, es decir, cómo los estudiantes entienden y aplican prácticas de investigación para el diseño. Las experiencias y actitudes de los estudiantes hacia la investigación, que se expresan en sus narrativas personales, en los procesos personales de diseño, en aspectos comunes y divergentes de los estilos para aprender. La investigación para el diseño está presente en los argumentos y explicaciones del proceso creativo de los estudiantes, en cómo los estudiantes articulan su comprensión de los problemas de diseño y las soluciones que desarrollan, y diversos tipos de habilidades que promueven la práctica de la investigación para el diseño (Lupton, 2011a; Shea, 2012; Stickdorn y Schneider, 2011) como el pensamiento crítico, el pensamiento innovador, el trabajo colaborativo, las habilidades de comunicación, las habilidades artísticas y la creatividad para la resolución de problemas.

Igualmente importante es, comprender el nuevo contexto en el que se desarrolla la enseñanza y el aprendizaje a distancia en el confinamiento que experimentamos todos, estudiantes y educadores. ¿Cuál es la dinámica que está surgiendo en la educación en línea? ¿Cómo es que este ambiente contribuye a la comprensión y aplicación de la investigación para el diseño? ¿Cómo las nuevas plataformas educativas apoyan a la investigación para el diseño? ¿Cuál es el impacto de la educación a distancia y las nuevas tecnologías en la formación de los estudiantes? ¿Qué tipo de métodos de instrucción se utilizan en la enseñanza de la investigación en este nuevo contexto? ¿Cuál es la conexión de la investigación con la nueva realidad? 


\section{Diseño gráfico e investigación}

Los procesos creativos del diseño gráfico crean sistemas de información que combinan texto, símbolos e imágenes profundamente entrelazados que contribuyen a mejorar las interacciones de las personas. Por lo que definimos el diseño gráfico como la disciplina que desarrolla sistemas de comunicación visual para facilitar las actividades humanas. Un diseñador gráfico puede combinar color, tipografía e imagen empleando una variedad de tecnologías para difundir información en medios visuales tanto materiales como digitales para contribuir en la solución de cualquier tipo de problema.

El Manifiesto de Educación del Diseño Gráfico de Icograda 2011 (Bennet y Vulpinari, 2011) expresa la necesidad de un desarrollo integral de las habilidades de los estudiantes frente a la evolución de los escenarios, cada vez más complejos que la nueva normalidad está presentando y sitúa la investigación como una de las actividades más importantes en la promoción del perfil adecuado de los diseñadores para enfrentar los requerimientos emergentes de la sociedad. Esta actividad se aprende y consolida en los talleres de diseño Los programas de diseño gráfico de pregrado en las universidades brindan cursos prácticos con un formato de estudio/laboratorio o taller de diseño, que es el modelo educativo de la mayoría de las escuelas de diseño (Kurt, 2009). Los cursos generalmente combinan el aprendizaje basado en la solución de problemas y el aprendizaje basado en proyectos de diseño. A través de estos talleres, el conocimiento de cursos teóricos se sintetiza y aplica en los procesos de diseño de los estudiantes. Los profesores e instructores suelen proporcionar los proyectos o problemas a resolver, y las líneas generales de las soluciones. En los talleres de diseño, el tiempo se estructura para proporcionar instrucción y asistencia individualizadas, así como la supervisión de la práctica de investigación para los proyectos a lo largo del proceso de diseño y el aprendizaje se consolida.

La investigación para el diseño y el proceso de diseño son actividades entrelazadas que se alimentan mutuamente en un proceso de gestión de la información y la toma de decisiones. La práctica de investigación para el diseño gráfico puede tener un impacto tanto en el proceso de diseño como en el producto. Press y Cooper (2003) presentan tres propósitos principales de la investigación para el diseño: Uno es la búsqueda de comprensión, que incluye la recopilación de datos sobre el mercado, el producto, las partes interesadas, los fabricantes, los proveedores, los clientes, los usuarios y su entorno. Dos, es la búsqueda de ideas a través de diversas técnicas de generación creativa de ideas. Y tres es, evaluar soluciones, que implican revisar diseños anteriores, probar las ideas y la tecnología, procesos y materiales necesarios, así como estrategias de marketing. 


\section{Investigación e intuición}

La investigación proporciona los datos necesarios para la toma de decisiones en el proceso de diseño en un proceso multidimensional, que implica, por un lado, la racionalidad, mantenerse objetivo, no emocional, no religioso, no instintivo, no moral, no cultural, y basado en la información disponible, acciones basadas en la experiencia. Por otro lado, la intuición, que es subjetiva, emocional, involucra el instinto, la cultura, y se basa en la imaginación y las experiencias (Eisenhardt y Zbaracki, 1992). Algunas situaciones permiten más racionalidad y otras requieren más intuición en el ámbito del desempeño humano; por lo tanto, la intuición y la racionalidad no son mutuamente excluyentes.

La toma de decisiones integra el pensamiento analítico e intuitivo en procesos complementarios e iterativos en los que uno u otro pueden prevalecer (Burke y Miller, 1999; Sinclair y Ashkanasy, 2005). Además, los estudios contemporáneos muestran cómo la intuición es cada vez más aceptada como parte de los caminos que las personas siguen en la toma de decisiones. El proceso intuitivo no es un razonamiento consciente, procesa información de manera integral y con frecuencia involucra emociones (Sinclair y Ashkanasy, 2005). Esos hechos llevan la intuición a expresiones no verbales utilizando imágenes y metáforas (Crossan, Lane y White, 1999), que es el lenguaje común de los diseñadores. Además, la intuición se caracteriza por su "naturaleza no lineal y no secuencial del procesamiento holístico" en la toma de decisiones (Sinclair y Ashkanazy, 2005), que se aplica a través del proceso de diseño iterativo. Algunos de los beneficios de la intuición en la toma de decisiones son: decisiones aceleradas, decisiones finales mejoradas, facilitar el desarrollo personal y decisiones compatibles con la cultura de la empresa (Burke y Miller, 1999). El contexto actual demanda un nivel cada vez más alto de agilidad en la toma de decisiones. Se requiere de decisiones más rápidas, más acertivas y más creativas. Por lo que es necesario entender la intuición como herramienta que apoya a la investigación y al proceso de diseño en tiempos de pandemia.

\section{Investigación y el proceso de diseño}

En esta investigación Sobre la base del modelo de seis etapas utilizado, se ha explorado con más detalle la diversidad de actividades de investigación que se desarrollan en el proceso de diseño (Valdovinos, 2016). La siguiente descripción de cada etapa integra la comprensión de los seis tipos de investigación para el diseño utilizados en el proceso de diseño y que pueden ayudar a entender el contexto contemporáneo (ver Tabla 1). 
Tabla 1. El proceso de diseño y actividades específicas de investigación.

\begin{tabular}{|c|c|c|c|c|}
\hline Etapa & Objetivo & Enfoque & Método & Características \\
\hline $\begin{array}{c}1 . \\
\text { Exploración }\end{array}$ & $\begin{array}{c}\text { Comprensió } \\
\text { n }\end{array}$ & $\begin{array}{c}\text { Mercado y } \\
\text { competencia } \\
\text { Estilo de } \\
\text { vida, entomo, } \\
\text { modas. } \\
\text { Antropología } \\
\text { y ethnografia }\end{array}$ & $\begin{array}{c}\text { Observación, revisión } \\
\text { de aspectos relevantes, } \\
\text { caminar por las calles, } \\
\text { toma de fotos, platicar } \\
\text { con la gente, lluvia de } \\
\text { ideas, mapas mentales, } \\
\text { entrevistas, grupos } \\
\text { focales, investigación } \\
\text { visual, exploración del } \\
\text { entomo }\end{array}$ & $\begin{array}{l}\text { Intuitiva, informal, y } \\
\text { formal, explorativa, } \\
\text { conectando con la } \\
\text { cultura de la } \\
\text { organización y su } \\
\text { entomo, con la } \\
\text { cultura del usuario y } \\
\text { su entomo }\end{array}$ \\
\hline $\begin{array}{c}2 . \\
\text { Definición }\end{array}$ & $\begin{array}{l}\text { Información } \\
\text { sobre la } \\
\text { transformac } \\
\text { ión } \\
\text { Generación } \\
\text { de } \\
\text { requerimien } \\
\text { tos } \\
\end{array}$ & $\begin{array}{l}\text { Centrado en } \\
\text { la práctica } \\
\text { Participatoria }\end{array}$ & $\begin{array}{c}\text { Identificación, } \\
\text { categorización, y } \\
\text { transformación en } \\
\text { categorías significativas } \\
\text { o cacaterísticas } \\
\text { significativas } \\
\text { requerimientos con } \\
\text { requerimientos } \\
\text { creativos }\end{array}$ & $\begin{array}{l}\text { Racional e intuitive, } \\
\text { formal, guiando el } \\
\text { criterio de diseño. }\end{array}$ \\
\hline $\begin{array}{c}3 . \\
\text { Creación }\end{array}$ & $\begin{array}{l}\text { Generación } \\
\text { de ideas }\end{array}$ & $\begin{array}{l}\text { Centrado en } \\
\text { la práctica } \\
\text { Participatoria }\end{array}$ & $\begin{array}{l}\text { Búsqueda de productos } \\
\text { anteriores o diseños; } \\
\text { tecnicas creativas y } \\
\text { bocetaje en un proceso } \\
\text { iterativo. }\end{array}$ & $\begin{array}{l}\text { Design thinking, } \\
\text { intuitivo, informal, } \\
\text { individuale } \\
\text { investigación } \\
\text { colectiva } \\
\end{array}$ \\
\hline $\begin{array}{c}4 . \\
\text { Desarrollo }\end{array}$ & $\begin{array}{l}\text { Evolución } \\
\text { de la idea }\end{array}$ & $\begin{array}{c}\text { Centrado en } \\
\text { la práctica } \\
\text { Tecnología } \\
\text { Participatoria } \\
\text { Contextual } \\
\text { Mercado } \\
\end{array}$ & $\begin{array}{l}\text { Prototipos, evaluación } \\
\text { de ideas, participación } \\
\text { de involucrados y la } \\
\text { audiencia; búsqueda de } \\
\text { tecnología, materiales y } \\
\text { procesos } \\
\end{array}$ & $\begin{array}{l}\text { Intuitiva pero más } \\
\text { formal y objetiva }\end{array}$ \\
\hline $\begin{array}{l}5 . \\
\text { Implementa } \\
\text { ción }\end{array}$ & $\begin{array}{c}\text { Producción } \\
\text { de la idea } \\
\begin{array}{c}\text { Presentació } \\
\text { n }\end{array} \\
\text { Difusión } \\
\end{array}$ & $\begin{array}{l}\text { Centrado en } \\
\text { la práctica } \\
\text { Tecnología } \\
\text { Participatoria } \\
\text { Contextual } \\
\text { Mercado }\end{array}$ & $\begin{array}{c}\text { Búsqueda de } \\
\text { tecnología, materiales y } \\
\text { procesos y la } \\
\text { transferencia de } \\
\text { métodos }\end{array}$ & $\begin{array}{c}\text { Formal e informal, } \\
\text { evaluación y } \\
\text { supervisión }\end{array}$ \\
\hline $\begin{array}{c}6 . \\
\text { Evaluación }\end{array}$ & $\begin{array}{l}\text { Impacto y } \\
\text { resultados }\end{array}$ & $\begin{array}{l}\text { Contextual, } \\
\text { Mercado, } \\
\text { modas, } \\
\text { conocimiento } \\
\text { de diseño } \\
\end{array}$ & $\begin{array}{c}\text { Cuantitativa y } \\
\text { cualitativa: medir y } \\
\text { evaluar resultados e } \\
\text { impacto }\end{array}$ & $\begin{array}{c}\text { Formal e informal, } \\
\text { deductive e intuitivo, } \\
\text { subjectivo y } \\
\text { objectivo. }\end{array}$ \\
\hline
\end{tabular}


Tabla 2. Seis etapas del proceso de diseño y sus actividades de investigación. (Diseño del autor)

1. Exploración y análisis general del caso. El propósito de esta etapa es crear una visión general sobre el escenario y sus actores de una manera intuitiva, y sentar las bases para las siguientes etapas. Las actividades involucradas en esta etapa temprana son: identificar la filosofía, el espíritu, los objetivos y las perspectivas de la organización para proporcionar una declaración general del problema; identificar el contexto; identificar a los individuos, hechos y elementos involucrados; y con esta información desarrollar un plan de acción (Lupton, 2011b). El estudiante debe identificar la necesidad de información sobre el caso, aplicar los métodos de investigación para adquirir esa información (Lupton, 2011b). Algunos ejemplos de métodos de investigación para esta etapa son: discusiones en línea, recorrer el lugar del usuario, etnografía rápida con video, conversación con las personas involucradas y evaluación del producto, toma de fotografías y su análisis, elaborar lista de términos empleados por el usuario, entre otros.

2. Definición o análisis específico del problema. El propósito de esta etapa es identificar, comprender y describir el problema. Así como establecer los requerimientos de manera integral y detallada. Los resultados de esta etapa deben ser: La descripción de los contextos sociales, culturales, económicos, educativos y físicos; definición de la audiencia que incluye: descripción de características demográficas (edad, género, estado civil, educación, ingresos, etc.) y características psicográficas (intereses, deseos, valores, metas, etc.); la declaración del problema y los requisitos de comunicación. El análisis puede mostrar diferentes causas del problema y conducir a soluciones integradas por diversas acciones o estrategia en las que el diseño gráfico es una parte de esas soluciones (Press \& Cooper, 2003). Esta etapa es importante promover entre los estudiantes en un trabajo de investigación colaborativo y con otras disciplinas para llegar a visiones y perspectivas interdisciplinarias integrales.

3. Creación o producción de ideas. El propósito de esta etapa es crear ideas, conceptos y soluciones. Este es un proceso divergente, es decir se abre al abanico de posibilidades. El diseñador produce tantas ideas como sea posible (Stickdorn y Schneider, 2011). Los principales resultados de esta etapa son las ideas preliminares. Las actividades involucradas en esta etapa son la aplicación de técnicas creativas tales como: lluvia de ideas, descarga visual del cerebro, conexiones forzadas, verbos de acción, todo desde todas partes; técnicas participativas como colaboración y codiseño (Lupton, 2011a; Lupton, 2011b). Las actividades de investigación relacionadas con esta etapa es la de exploración de proyectos similares ya terminados, en un trabajo de benchmarking y de inspiración.

4. Desarrollo de ideas. Esta es una etapa de proceso convergente, lo que significa reducir la cantidad de ideas mediante un proceso de evaluación y selección de las mejores propuestas (Stickdorn y Schneider, 2011). Los resultados de esta etapa son sólo algunas ideas seleccionadas y desarrolladas. Las actividades involucradas en esta etapa son: selección y, a 
veces, ideas mixtas; desarrollo de las ideas seleccionadas a través de un proceso iterativo de evaluar y corregir, volver a evaluar y corregir; centrándose en los detalles; incorporar tecnología, materiales y procesos; desarrollo de prototipos o maquetación para evaluación, involucrando a la organización y al usuario en un proceso democrático y participativo; desarrollo de elementos de diseño específicos y complementarios, tales como producción y distribución. En esta etapa la investigación es el núcleo del proceso de diseño empleando diversos métodos de evaluación de las propuestas.

5. Implementación. Es decir la gestión del diseño final para los procesos de producción y distribución. El propósito en esta etapa es asegurar la calidad de la producción y el seguimiento del lanzamiento de acuerdo al plan estratégico. Las actividades involucradas en esta etapa son: definición de las características finales del proyecto; definición de tecnología, materiales y procesos de producción y distribución; conectarse con técnicos y especialistas para hacer la transferencia adecuada del diseño a las plataformas de producción; realizar supervisión de la producción; evaluación del producto final; supervisión y evaluación de distribución. Para lograr los objetivos y tiempos, la investigación del equipo y tecnología de producción puede ser crítica.

6. Evaluación. Revisión del impacto y de la respuesta del usuario a nuestro diseño. El propósito es evaluar la respuesta del cliente y de las personas involucradas, como productores, técnicos, vendedores y usuarios, todos ellos proporcionan valiosa información. Esta etapa también representa la oportunidad para que el diseñador y la organización aprendan sobre todo el proceso de diseño y el conocimiento resultante. Las actividades involucradas en esta etapa son: evaluar cualitativamente y cuantitativamente los resultados, es decir cuantificar la diferencia del antes y el después, qué tanto se logró la realidad deseada. Se emplean técnicas como consultas, grupos focales, entrevistas, observación y visitas al lugar del problema.

Las diversas actividades de investigación para el diseño descritas a través de nuestro modelo de proceso de diseño proporcionan una plataforma de características específicas de la investigación para el diseño que pueden ser promovidas tanto en los talleres de diseño como en los cursos de investigación de diseño. Estas actividades deben ser empleadas para desarrollar técnicas de recopilación de datos creativas e innovadoras, con un principio de economía, que hagan esta actividad en el menor tiempo y costos para que los estudiantes realmente incorporen estas prácticas en sus procesos de diseño (Valdovinos, 2016), y adquieran las herramientas que les permitirá entender las nuevas actitudes, los nuevos valores, aspiraciones e intereses que han evolucionado en los meses que el mundo se recluyó en sus casas. 


\section{Constructivismo en la educación del diseño}

La perspectiva del constructivismo puede ayudar a comprender la instrucción de la investigación para el diseño en los talleres de diseño, donde las estrategias de enseñanza de la educación del diseño pueden explicarse por la teoría del aprendizaje a través de la experiencia de Dewey, quien creía en este tipo de interacción en el aula como comunidad para compartir experiencias y acciones, ya que con un proyecto común en mente, mejora la experiencia de aprendizaje (Dewey 1916, p. 75).

En este sentido Kurt (2009) explica que los talleres de diseño "ofrecen un excelente ejemplo de un entorno de enseñanza colaborativo, multisensorial, centrado en el alumno, constructivista y basado en problemas experimentales" (p. 403). La suposición fundamental del constructivismo es que "las personas crean conocimiento a partir de la interacción entre sus conocimientos o creencias existentes y las nuevas ideas o situaciones que encuentran" (Airasian y Walsh, 1997, p. 2). Por lo que resulta necesario como educadores promover esa interacción, empleando estrategias didácticas que acerquen los estudiantes a los usuarios y sus contextos. Para Vigotsky (1978), la experiencia del individuo que permite expandir la construcción de nuevos conocimientos, llega de manera significativa a través de la interacción con los demás. (Pritchard y Wollard, 2010).

Además, Bruner (1977) enfatizó la noción de aprendizaje como un proceso activo y social que es relevante para este estudio y para la comprensión del aprendizaje dentro del proceso de diseño. En este sentido, Bruner explica cómo el individuo da sentido a las experiencias y expande su esquema al seleccionar, analizar, transformar, crear hipótesis y tomar decisiones basadas en construcciones anteriores (Bruner, 1977; Pritchard y Wollard, 2010). Este concepto ilumina el papel relevante del educador, quien puede promover la interacción de los estudiantes, con el profesor, con la sociedad y con el medio ambiente lo que resulta fundamental para entender el nuevo contexto y sus demandas. La interacción con el ususario y su entorno, fruto de la aproximación que permite la investigación para el diseño va a transfomar la visión de los estudiantes para convertirlos en agentes de cambio ante el nuevo contexto en el mundo.

\section{Conclusión}

Este capítulo ha explorado la investigación para el diseño en contextos contemporáneos y los desafíos en la educación del diseño que deben responder a los cambios en la comunicación, la tecnología y sobre todo en la cultura.

El proceso de diseño se ha descrito con un modelo que facilita la identificación de diversos tipos de prácticas de investigación que intervienen en cada una de sus seis etapas. La enseñanza de la investigación para el diseño debe considerar técnicas informales y formales de recopilación de datos e incluir la intuición; debe ser flexible y utilizar una amplia variedad de métodos con un criterio de economía. 
El enfoque constructivista nos ayuda a entender los procesos de aprendizaje del diseño y de la investigación que involucran la socialización y el acercamiento al usuario, al contexto y a todos los involucrados para actualizar las prácticas de enseñanza y las competencias que el nuevo entorno demanda.

La educación en diseño debe desarrollar estrategias que promuevan la investigación en todas sus formas para alimentar todo el proceso de diseño. Esta investigación desarrollará habilidades como la observación, pensamiento crítico, la comprensión del problema, la generación de ideas, el juicio reflexivo, la toma de decisiones, las actitudes y disposiciones para evaluar los resultados. Todas estas habilidades resultan básicas para enfrentar el aspecto evolutivo del nuevo contexto. La investigación para el diseño tendrá un papel definitivo si se actualiza y desarrolla en la formación de diseñadores que logren enfrentar con éxito los retos del nuevo entorno que presenta la pandemia del Covid-19.

\section{Listado de Referencia Bibliográfica}

Airasian, P. W.; Walsh, M. E. (1997). Constructivist Cautions. En Phi Delta Kappan, Vol. 78. Bennet, A. G. y Vulpinari, O. (Eds.) (2011). ICOGRADA Manifiesto de educación del diseño gráfico 2011. Italia: ICOGRADA.

Bruner, J. S. (1977). "Early social interaction and language acquisition". In H. R. Schaffer (ed.) Studies in Mother-infant Interaction (pp. 271-289). London: Academic Press.

Burke, Lisa A. y Miller, Monica K. (1999). Taking the Mystery out of Intuitive Decision Making. En The Academy of Management Executive (1993-2005), 13(4), 91-99. Consultado en http://www.jstor.org/stable/4165589 Abril 26, 2021.

Crossan, M. M., Lane, H. W. and White, R. E. (1999) An organizational learning framework: From intuition to institution. En Academy of Management Review, Vol 24, No. 3, pp 522-537. (13) (PDF) From Individual Learning to Organizational Learning. Available from: https://www.researchgate.net/publication/252380753_From_Individual_Learning_to_Organizational_Learning [accessed Apr 26 2021].

Dewey, John. (1916). Democracy and education, New York: Macmillan.

Eisenhardt, Kathleen M., and Mark J. Zbaracki. "Strategic Decision Making." En Strategic Management Journal, vol. 13, 1992, pp. 17-37. JSTOR, www.jstor.org/stable/2486364. Accessed 26 Apr. 2021.

Lupton, Ellen. (ed.). (2011a). Graphic design thinking: beyond brainstorming. New York: Princeton Architectural Press.

Lupton, Ellen. (2011b). Foreword. In Armstrong H. \& Stojmirovic, Z. Participate designing with user-generated content. New York: Princeton Architectural Press.

Macías, Luis, Valdovinos, Salvador y Rogel, Erika. (2016). Educación para un diseño sostenible y por competencias. En Taller Servicio 24 horas, Año 11 / Num. 22 / Sept 2015-Feb 2016. México: UAM Azcapotzalco.

Press, Mike y Rachel Cooper. El diseño como experiencia: El papel del diseño y los diseñadores en el siglo XXI. Barcelona: Gustavo Gili, 2007. 
Pritchard, Alan y Woollard, John (2010) Psychology for the classroom: constructivism and social learning. En Research Gate Consultado en https://www.researchgate.net/publication/313214018_Psychology_for_the_classroom_constructivism_and_social_learning Shaughnessy, A. (2010). How to be a graphic designer without losing your soul. New York: Laurence King.

Shea, A. (2012). Designing for Social Change. Strategies for Community-Based Graphic Design. New York: Princeton Architectural Press.

Sinclair, Martha y Ashkanasy, Neal (2005) Intuition: Myth or a Decision-Making tool? En Management Learning. Septiembre 2005 Research Gate.

Stickdorn, Marc y Schneider, Jakob. (2012). This is service design thinking: Basic, tools, cases. New York: Princeton Architectural Press

Valdovinos, Salvador. (2016) “A Comparative Study of Research-for-design: Teaching and Learning in Two Undergraduate Graphic Design Programs in Canada and Mexico”. Tesis de doctorado, Universidad Concordia.

Valdovinos, Salvador y Rogel, Erika. (2021). El Diseño Gráfico en la Industria: Competencias para la nueva realidad, después del Covid-19. En LEGADO de Arquitectura y Diseño. No. 28, Julio-Diciembre 2020.

Vygotsky, Lev S. (1978). Mind in society: The Development of higher psychological processes. M. Cole, V. John-Steiner, S. Scribner, \& E. Souberman (Eds.) Cambridge, MA: Harvard University Press.

\begin{abstract}
This project explores the field of research for design as one of the activities that graphic designers can train with the knowledge, skills and attitudes to help understand the new context and facilitate human activities in adapting to the new reality, new culture, new ways of doing things and new connections between objects and their meanings. Research must be learned with didactic strategies that bring students closer to the user and their context so that they acquire an updated and accurate vision of the evolution of society.
\end{abstract}

Keywords: Research for design - research teaching - Covid-19 - design process - design workshops.

Resumo: Este projeto explora o campo da pesquisa para o design como uma das atividades que os designers gráficos podem formar com os conhecimentos, habilidades e atitudes para ajudar a compreender o novo contexto e facilitar as atividades humanas na adaptação à nova realidade, nova cultura., Novas formas de fazer coisas e novas conexões entre objetos e seus significados. A pesquisa deve ser aprendida com estratégias didáticas que aproximem o aluno do usuário e de seu contexto, para que tenha uma visão atualizada e precisa da evolução da sociedade. 
Palavras chave: Pesquisa para design - ensino de pesquisa - Covid-19 - processo de design - workshops de design.

[Las traducciones de los abstracts fueron supervisadas por el autor de cada artículo] 